Z. klin. Chem. u. klin. Biochem.

8. Jg., S. 149-155, März 1970

\title{
Zur Frage der Identität von Glutathionperoxidase aus Erythrocyten und Leber („Contraction factor I“) der Ratte
}

\author{
Von L. Flohé, W. Schlegel und E. Schatch
}

\begin{abstract}
Aus dem Institut für Pbysiologische Chenie und Biocbemie der Universität Tiibingen (Direktor: Prof. Dr. Dr. G. Weitzel)
\end{abstract}
(Eingegangen am 25. November 1969)

\begin{abstract}
Glutathionperoxidase (Glutathion: $\mathrm{H}_{2} \mathrm{O}_{2}$ Oxidoreduktasc, EC 1.11.1.9) wird 7500 fach aus Erythrocyten und etwa 200 fach aus der Leber der Ratte angereichert. Die Enzympräparate enthalten keine Verunreinigungen, dic störende Nebenreaktionen hervorrufen. Vergleichende Untersuchungen ergeben in folgenden Punkten keinen signifikanten Unterschicd zwischen Leber- und Erythrocytenenzym: 1. Chromatographie auf Sephadex G 150; 2. Elektrophorese auf Celluloseacetatfolien bei pH 5,5-8,0 bei verschiedenen Ionenstärken; 3. Stabilität; 4. Donorsubstratspezifität; 5. Michaelis-Konstante für $\mathrm{H}_{2} \mathrm{O}_{2}$; 6. Reaktionskinetik erster Ordnung in bezug auf GSH. Die Ergebnisse bestätigen die Vermutung, daß Glutathionperoxidase aus Erythrocyten mit dem entsprechenden Enzym der Leber („Contraction factor $I^{\prime c)}$ ) identisch ist. Aufgrund dieses Identitätsnachweises ergibt sich die Möglichkeit, die an der Glutathionperoxidase der Leber gewonnenen Erkenntnisse für die Diskussion der biologischen Funktion des Enzyms in den Erythrocyten nutzbar zu machen.
\end{abstract}

\section{The identity of glutatbione peroxidase from erytbrocytes and liver (contraction factor I) of the rat}

Glutathione peroxidase (glutathione: $\mathrm{H}_{2} \mathrm{O}_{2}$ oxidoreductase, EC 1.11.1.9) was purified 7500 fold from rat exythrocytes and approximately 200 fold from rat liver. The enzyme preparations do not contain impurities which result in interfering reactions. Comparative investigations of enzyme from both liver and erythrocytes do not reveal significant differences in the following points: 1 . chromatography on Sephadex G 150; electrophoresis on cellulose acetate strips at $\mathrm{pH} 5.5-8.0$ and varying ionic strength; 3 . stability; 4 . specificity for the donor substrate; 5. Michaelis constant for $\mathrm{H}_{2} \mathrm{O}_{2} ; 6$. first order kinetics with regard to GSH. The results confirm the hypothesis that glutathione peroxidase from erythrocytes is identical with the liver enzyme ("contraction factor I"). In view of the established identity it is possible to discuss the biological function of GSH peroxidase in erythrocytes in the light of the numerous results obtained with the liver enzyme.

Von Neubert, Wojtczak und Lehninger (1) wurde 1962 ein hitzelabiler Faktor beschrieben, der bei Zugabe von ATP und $\mathrm{Mg}^{++}$eine Kontraktion von Mitochondrien ermöglichte, die durch Behandlung mit überphysiologischen Glutathionkonzentrationen eine Schwellung erlitten hatten. Dieser "Contraction factor I" (CFaktor I) findet sich sowohl in der löslichen Fraktion der Rattenleberzellen als auch strukturgebunden an der Mitochondrienmembran. Durch Ultraschall oder hohe Glutathionkonzentrationen läß sich der strukturgebundene Anteil solubilisieren (1). In der lokalen Verminderung der Kontraktionsfaktorkonzentration an der Mitochondrienmembran bei hohen Glutathionkonzentrationen wird die Ursache des erwähnten Schwellungsvorganges gesehen. In l. c. (1) wird weiter gezeigt, da $B$ Kontraktionsfaktor I Glutathionperoxidase-Aktivität besitzt. Da C-Faktor I sich zudem auf DEAE-Cellulose ähnlich verhält und ebenso labil ist wie die 1957 von MrLls erstmals beschriebene Glutathionperoxidase aus Rindererythrocyten (2), wird eine Identität von CFaktor I mit GSH: $\mathrm{H}_{2} \mathrm{O}_{2}$-Oxidoreduktase (GSH-POD) angenommen (1).

Die biologische Funktion der Glutathionperoxidase wird allerdings in Leber und Blut recht unterschiedlich beurteilt. Zwar fand man die C-Faktor-Aktivität schließlich mit der Peroxidaseaktivität kausal verknüpft: Der Arbeitskreis von HuNTER (3-G) konnte zeigen, daß gerade die durch GSH, Ascorbat und Eisenionen hervorgerufene Schwellung der Mitochondrien von der
Bildung von Lipidperoxiden in der Mitochondrienmembran begleitet und wahrscheinlich ursächlich bedingt ist. Aus zahlreichen Untersuchungen von LitTLE und O'Brien (7-9) und Christophersen $(10,11)$ geht hervor, daß eben diese Hydroperoxide ungesättigter Fettsäuren durch Glutathionperoxidase reduziert werden können. Obwohl auch der $\mathrm{H}_{2} \mathrm{O}_{2}$-Beseitigung durch GSH-Peroxidase in der Leber eine gewisse Bedeutung zukommt (12), wird in diesem Organ die Erhaltung der Mitochondrienmembran in funktionstüchtigem $\mathrm{Zu}$ stand als eigentliche Aufgabe des Enzyms betrachtet. Demgegenüber wird für die Erythrocyten bislang ausschließlich die $\mathrm{H}_{2} \mathrm{O}_{2}$-Elimination als Funktion der Glutathionperoxidase diskutiert. So konnten CoHEN und HochsteIN (13) am Ganzzellsystem zeigen, daß der Glutathionperoxidase bei $\mathrm{H}_{2} \mathrm{O}_{2}$-Konzentrationen unter $3 \mu \mathrm{M}$ eine größere Bedeutung zukommt als der Katalase. Die Hypothese konnte unlängst in unserem Arbeitskreis $(14,15)$ durch kinetische Untersuchungen mit hochgereinigter GSH-Peroxidase aus Rindererythrocyten gefestigt werden. Obwohl das Blutenzym ebenfalls Linolsäurehydroperoxid umsetzt (16), fand dieser Stoffwechselweg bei der Beurteilung der Peroxidasefunktion der Erythrocyten keine Beachtung.

Abgesehen von diesen Kontroversen in der Diskussion der physiologischen Funktion der GSH-POD in Blut und Leber finden sich in der Literatur einige Angaben zur Stabilität (17), Kinetik $(12,18)$ und Substratspezifität (8) der Leber-GSH-Peroxidase, die sowohl den 
Milusschen Beobachtungen (19) als auch unseren Erfahrungen mit der GSH-POD aus Rindererythrocyten widersprechen (20).

Glutathionperoxidase ist zur Zeit nicht in ausreichender Menge und Reinheit zugänglich, um eindeutige chemische Identitätsbeweise zu ermöglichen. Wir haben daher zur Prüfung einer eventuellen Organspezifität GSH-POD aus Erythrocyten und Leber der Ratte mit der gleichen Methode (20) angereichert. Mit den Ptäparaten, die zumindest frei von störenden Verunreinigungen waren, wurde Kinetik, Donor-Substratspezifität und elektrophoretisches Verhalten geprüft.

\section{Material und Methoden}

\section{Reagenzien}

Anorganische Reagenzien, Substanzen „pro analysi“ der Firma Merck, Darmstadt.

Heparin (Liquemin), Hoffmann La Roche, Grenzach/Baden; Glutatbion (GSH), Hoffmann La Roche, Grenzach/Baden; Merck, Darmstadt; Boehringer, Mannheim; Zellstoffwerk Waldhof, Mannheim; Glutathionreduletase aus Hefe, NADPH und Meerrettichperoxydase, Boehringer, Mannheim; Scopoletin und Sucrose, Mann Research Laboratories, New York; Sephadex G 50 coarse, G 150 und DEAE-Sephadex A 50, Pharmacia, Uppsala; Cj'steamin $\mathrm{HCl}$ und L-Cystein $\mathrm{HCl}$ p. a., Merck, Darmstadt; frisch destillierter Mercaptoessigsäuremetbjlester und $\beta$-Mercaptopropionsäuremetbylester wurden uns dankenswerterweise von Dr. G. JuNG (Institut für Organische Chemie der Universität Tübingen) zur Verfügung gestellt.

\section{Präparate}

$\gamma$-L-Glutamyl-L-Cysteinmetbylester $\mathrm{HCl}$

a) N-t-BOC-Glutaminsäure- $\alpha-\mathrm{OBu}^{\mathrm{t}}-\gamma$-Benzyldiester (I):

$16,5 \mathrm{~g}\left(50 \mathrm{mMol}\right.$ L-Glutaminsäure- $\alpha-\mathrm{OBu}^{\mathrm{t}}-\gamma$-Benzyldiester $\cdot \mathrm{HCl}$ $(21,22)$ wurden nach der Methode von SchnABEL (23) mittels $11 \mathrm{~g}(75 \mathrm{mMol}) \mathrm{t}$-BOC-Azid bei $\mathrm{pH} 9,5$ in (I) überführt. Ausbeute: $15,7 \mathrm{~g}(80 \%)$; Öl.

b) N-t-BOC-Glutaminsäure- $\alpha-O B u^{t}$ (II):

$15,7 \mathrm{~g}$ (40 mMol) wurden in $100 \mathrm{~m} /$ Methanol mit Pd/Aktivkohle hydriert.

Ausbeute: $9,8 \mathrm{~g}(80 \%)$; Fp. 107-109 (Essigester/Petroläther) $\mathrm{C}_{14} \mathrm{H}_{25} \mathrm{NO}_{6}(303,4) \quad \mathrm{N}_{\text {ber }}=4,62 \% \quad \mathrm{~N}_{\text {ger }}=4,45 \%$

c) Bis N-t-BOC-Glutamyl- $\alpha$-OBut-Cystindimethylester (III):

$6 \mathrm{~g}(20 \mathrm{mMol})$ (II) wurden in $50 \mathrm{ml}$ Chloroform gelöst, $3,41 \mathrm{~g}$ (10 mMol) L-Cystindimethylester $\cdot 2 \mathrm{HCl}$ (24) und $2,8 \mathrm{ml}$ (20 mMol) Triäthylamin zugefügt und bei $-15^{\circ} \mathrm{mit} 4,2 \mathrm{~g}(20 \mathrm{mMol})$ DCCI in $20 \mathrm{ml}$ Chloroform versetzt. Die übliche Aufarbeitung ergab nach Umkristallisation aus Essigester/Petroläther $5 \mathrm{~g} \mathrm{(60 \% )}$ (III); Fp. 110-112

$\mathrm{C}_{36} \mathrm{H}_{62} \mathrm{~N}_{1} \mathrm{O}_{14} \mathrm{~S}_{2}(838,0) \quad \mathrm{N}_{\text {ber }}=6,68 \% \mathrm{~N}_{\text {ger }}=6,78 \%$

$$
\begin{array}{ll}
N_{\text {ber }}=6,68 \% & N_{\text {gef }}=6,78 \% \\
S_{\text {ber }}=7,64 \% & S_{\text {ger }}=7,35 \%
\end{array}
$$

d) Bis- $\gamma$-Glutamyl-Cystindimethylester $\cdot 2 \mathrm{HCl}$ (IV):

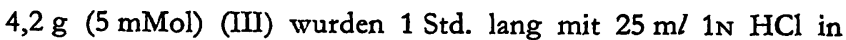
Eisessig behandelt, dann wurde auf $10 \mathrm{ml}$ eingeengt und mit absol. Äther gefällt.

Ausbeute: $2,7 \mathrm{~g}(90 \%)$; hygroskopisch.

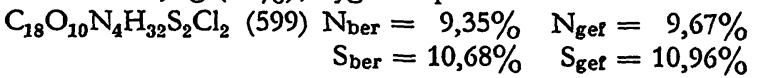

e) $\gamma$-Glutamyl-Cysteinmethylester $\cdot \mathrm{HCl}$ :

$1,5 \mathrm{~g}(2,5 \mathrm{mMol})$ (IV) wurden mit $10 \mathrm{ml}$ Mercaptoäthanol $5 \mathrm{Stdn}$. bei $50^{\circ}$ behandelt, anschließend wurde mit absol. Ather gefällt, zentrifugiert und sorgfältig mit Äther gewaschen und über $\mathrm{P}_{2} \mathrm{O}_{5}$ getrocknet.

Ausbeute: $1,5 \mathrm{~g}$ (quantitativ); $\mathrm{SH}-\mathrm{Gehalt}$ (polarographisch bestimmt) $>90 \%$.
Glutathionperoxidase aus Rattenerytbrocyten wurde im wesentlichen nach 1. c. (20) angereichert. Da sich der IP der GSH-POD der Ratte von dem des Rinderenzyms unterscheidet (s. u. und l. c. (25)), ergaben sich Schwierigkeiten bei der Austauscherchromatographie. Die Aufarbeitung soll daher kurz geschildert werden: 50 SIV-50Ratten wurden nach Dekapitation entblutet. Die Erythrocyten wurden $4 \mathrm{mal}$ mit 0,9 proz. $\mathrm{NaCl}$-Lösung gewaschen und durch halbstündiges Schütteln mit $0,5 \mathrm{Vol} \mathrm{H}_{2} \mathrm{O}$ und $0,3 \mathrm{Vol} \mathrm{CCl}_{4}$ hämolysiert. Nach Zentrifugation bei $2000 \mathrm{~g}$ wurde das stromafreie Hämolysat abgehebert (F1) und durch Chromatographie über Sephadex $G 50$ coarse in einen $0,005 \mathrm{M}$ Kaliumphosphatpuffer $\mathrm{pH} 7,3$ überführt (F 2). Die nun geplante Adsorption an DEAE-Sephadex A 50 (vgl. 1.c. (20)) gelang nicht. Für die $300 \mathrm{ml}$ F 2 reichte die Kapazität der DEAE-Sephadexsäule $(700 \mathrm{ml}$ Bettvolumen; 0,005M K-Phosphat $\mathrm{pH} \mathrm{7,3)}$ wider Erwarten nicht aus; nahezu die gesamte Aktivität fand sich in Vorlauf und Waschwasser. Wir fällten daher das Enzym aus den vereinigten aktiven Fraktionen (F 3) zusammen mit Hämoglobin bei $70 \%\left(\mathrm{NH}_{4}\right)_{2} \mathrm{SO}_{4}$-Sättigung aus. Der Niederschlag wurde in wenig Wasser aufgenommen (F 4) und auf eine Sephadex G 150 Säule $(4 \times 200 \mathrm{~cm} ; 0,005 \mathrm{M}$ K-Phosphat $\mathrm{pH} 7,3)$ gegeben. Die aktiven Fraktionen des Eluats (F 5) wurden auf DEAE-Sephadex (Säulenabmessungen $4 \times 25 \mathrm{~cm} ; 0,005 \mathrm{M}$ K-Phospbat $\mathrm{pH} 7,3$ ) aufgetragen und mit einem Phosphatpuffer-Gradienten $z$ wischen 0,005 und $0,06 \mathrm{M}$ eluiert (F 6). Arbeitstemperatur durchgehend $4^{\circ}$ (vgl. Tab. 1).

\section{GSH-Peroxidase aus Rattenleber}

$250 \mathrm{~g}$ frisch entnommene Leber von 50 SIV-50-Ratten wurde sofort in eisgekühlte $0,25 \mathrm{M}$ Sucrose in $0,02 \mathrm{M}$ Tris-Acetat-Puffer $\mathrm{pH} 7,6$ gegeben. Das Lebergewebe wurde unter Zugabe von $3 \mathrm{ml}$ Sucroselösung (s. o.) pro g Leber 6-8 Min. lang bei 200 U./Min. in einem Potter-Elvehjem-Homogenisator mit Teflonpistill homogenisiert. Die lösliche Zellfraktion wurde durch Zentrifugation in der Beckman-Spinco-Ultrazentrifuge bei $48000 \mathrm{~g}$ ( $90 \mathrm{Min}$.) gewonnen. Die Aufarbeitung dieser Fraktion (F 1) verlief wie oben angegeben; nur wurde bei der ersten DEAE-Sephadexchromatographie ein nunmehr austeichendes Bettrolumen von 2,0 l eingesetzt. Die Elution erfolgte mit einem $0,06 \mathrm{M}$ K-Phosphatpuffer $\mathrm{pH} 7,0$ (F 3), die anschließende $\left(\mathrm{NH}_{4}\right)_{2} \mathrm{SO}_{4}$-Fällung bei $55 \%$ Sättigung (vgl. Tab. 2). Proteinbestimmungen wurden nach LOWRY und Mitarbeitern (26), Hämoglobinbestimmungen nach THAUER und Mitarbeitern (27) durchgeführt. Auf Katalaseaktivität wurde mit dem Scopoletintest nach AEBI (28) geprüft. Glutathionperoxidaseaktivität wurde nach 1 . c. (20) bestimmt.

\section{Kinetik}

Die Abhängigkeit der GSH-Peroxidaseaktivität von der GSHKonzentration wurde mit Glutathionpräparaten verschiedener Firmen mit Hilfe des polarographischen Testes (20) untersucht. Die Bestimmung der Michaeliskonstante für $\mathrm{H}_{2} \mathrm{O}_{2}$ erfolgte nach l. c. (14).

\section{Donorsubstratspezifität}

Die Reaktivität der GSH-Peroxidase mit verschiedenen SH-Verbindungen wurde analog zur polarographischen Aktivitätsbestimmung geprüft. Die Konzentrationen der SH-Verbindungen wurden mit Hilfe von Eichkurven ermittelt.

\section{Elektrophorese}

Die elektrophoretische Beweglichkeit der GSH-Peroxidase aus Rattenblut und -leber wurde in der Beckman-Mikrozonen-Elektrophorese auf Cellogelstreifen in Tris-Maleat-Puffer über einen pH-Bereich von 5,5-8,0 untersucht (nach FroHé (25)). Blutund Leberenzym wurden jeweils nebeneinander auf dem gleichen Streifen aufgetragen. Die Enzymaktivität auf dem Elektrophoresestreifen wurde nach l. c. (25) sichtbar gemacht.

\section{Ergebnisse}

Das zur Anreicherung der Glutathionperoxidase aus Erythrocyten und Leber der Ratte verwandte Präpara- 
Tab. 1

Anreicherung der Glutathionperoxidase aus Rattenerythrocyten

\begin{tabular}{|c|c|c|c|c|c|c|c|}
\hline \multicolumn{2}{|c|}{ Fraktion } & \multirow{2}{*}{$\begin{array}{r}\text { Vol ml } \\
140 \\
300 \\
6000 \\
30 \\
250 \\
225\end{array}$} & \multirow{2}{*}{$\frac{\text { Protein mg }}{24500,0}$} & \multirow{2}{*}{$\frac{\text { Aktivitat } U}{1540}$} & \multirow{2}{*}{$\frac{\text { Spez. Akt. U/mg }}{0,063}$} & \multirow{2}{*}{$\frac{\text { Anreicherung }}{0}$} & \multirow{2}{*}{$\frac{\text { Ausbeute \% }}{100}$} \\
\hline $\begin{array}{l}\text { F1 } \\
\text { F2 } \\
\text { F3 } \\
\text { F4 } \\
\text { F5 } \\
\text { F6 }\end{array}$ & $\begin{array}{l}\text { Stromafreies Hämolysat } \\
\text { Sephadex G-50 } \\
\text { 1. DEAE-Sephadex A-50 } \\
\text { (NH, } \text { I }_{2} \text { SO, Fällung } 70 \% \\
\text { Sephadex G-150 } \\
\text { 2. DEAE-Sephadex A-50 }\end{array}$ & & & & & & \\
\hline
\end{tabular}

Tab. 2

Anreicherung der Glutathionperoxidase aus Leberhomogenat der Ratte

\begin{tabular}{|c|c|c|c|c|c|c|c|}
\hline \multicolumn{2}{|c|}{ Fraktion } & \multirow{3}{*}{$\begin{array}{r}\text { Vol ml } \\
660 \\
1500 \\
6000 \\
20 \\
550 \\
275\end{array}$} & \multirow{2}{*}{$\begin{array}{c}\text { Protein } \mathrm{mg} \\
75000 \\
52500\end{array}$} & \multirow{2}{*}{ 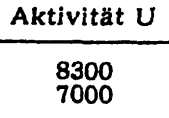 } & \multirow{2}{*}{ 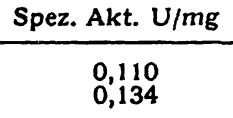 } & \multirow{2}{*}{ 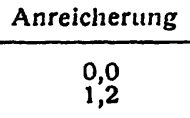 } & \multirow{2}{*}{$\begin{array}{c}\text { Ausbeute \% } \\
100,0 \\
84,5\end{array}$} \\
\hline $\begin{array}{l}\text { F1 } \\
\text { F2 }\end{array}$ & $\begin{array}{l}48000 \mathrm{~g} \text { Überstand } \\
\text { Sephadex G-50 }\end{array}$ & & & & & & \\
\hline $\begin{array}{l}\text { F4 } \\
\text { F5 } \\
\text { F6 }\end{array}$ & $\begin{array}{l}\left(\mathrm{NH}_{4}\right)_{2} \mathrm{SO}_{4} \text { Fällung } 55 \% \\
\text { Sephadex G-150 } \\
\text { 2. DEAE-Sephadex A-50 }\end{array}$ & & $\begin{array}{r}640 \\
165 \\
81\end{array}$ & $\begin{array}{l}3900 \\
2900 \\
1610\end{array}$ & $\begin{array}{r}6,100 \\
17,500 \\
20,000\end{array}$ & $\begin{array}{r}55,0 \\
160,0 \\
180,0\end{array}$ & $\begin{array}{l}47,0 \\
35,0 \\
19,5\end{array}$ \\
\hline
\end{tabular}

tionsschema war von uns (20) zur Isolierung des Enzyms aus Rindererythrocyten ausgearbeitet worden. Es zeigte sich jedoch, daß eine streng analoge Aufarbeitung des Enzyms aus der Ratte nicht möglich war. Da der IP des Enzyms bei der Ratte gegenüber dem Rinderenzym um $0,8 \mathrm{pH}$-Einheiten verschoben ist (s. u. und vgl. 1. c. (25)), wurde die GSH-Peroxidase des Rattenblutes auf der ersten DEAE-Säule nicht quantitativ adsorbiert. Wie Tabelle 1 zeigt, erhielten wir trotz dieses Zwischenfalls eine sehr hohe Anreicherung. Die Gegenwart von Hämoglobin und Katalase in F 6, die mit der Aktivitätsbestimmung der Glutathionperoxidase interferieren $(20,29,30)$, konnte ausgeschlossen werden.

Die Anreicherung der Glutathionperoxidase aus der Leber ist wesentlich problematischer. In Ubereinstimmung mit älteren Angaben (17) fanden wir in Leberhomogenaten einen ungewohnt schnellen Abfall der Glutathionperoxidase-Aktivität. Nach der ersten chromatographischen Trennung des Materials auf DEAESephadex jedoch wurden die Aktivitätsverluste geringer. Bei der schließlich erreichten Anreicherungsstufe (F 6) konnten wir keinen signifikanten Unterschied in der Stabilität von Blut- und Leberenzym mehr feststellen. Die Aktivitäten beider Chargen sanken im Verlauf von $2 \frac{1}{2}$ Monaten um nicht mehr als $40 \%$ ab. Die geringe Stabilität des Enzyms in der Leber scheint also nicht auf einer ausgeprägteren Denaturierungsneigung zu beruhen, sondern durch noch nicht identifizierte Komponenten des Leberhomogenats bedingt $z u$ sein. Wie aus Tabelle $2 \mathrm{zu}$ ersehen ist, erreichten wir bei weitem nicht die spezifische Akțivität der Erythrocytenpräparation. Immerhin konnten wir auch hier die Gegenwart von Katalase, Hämoglobin und Cytochromen ausschließen. Eine Störung der durchgeführten Untersuchungen durch die noch vorhandenen Proteinverunreinigungen ist somit auszuschließen.

Bei der Anreicherung verhielt sich die Glutathionperoxidase aus Erythrocyten und Leber völlig analog. Die Position der Aktivität bei der Chromatographie auf Sephadex G 150 läßt auf ein Molekulargewicht schlieBen, das dem der Erythrocyten-GSH-POD des Rindes ( 85000) (20) und des Menschen ( 100000) (29) vergleichbar ist.

\section{Kinetik}

Während für Glutathionperoxidase aus Rinderblut eine Reaktionskinetik erster Ordnung in bezug auf das Donorsubstrat in allen Konzentrationsbereichen als gesichert gelten kann $(7,10,14,15,20)$ (für das Enzym aus Rattenerythrocyten wurden bisher keine Daten veröffentlicht), wurden für das Rattenleberenzym $\mathrm{K}_{\mathrm{m}}$ (GSH)-Werte von $10 \mathrm{~mm}$ oder mehr (12) und $5 \mathrm{~mm}$ (18) angegeben. Littre und O'Brien $(7,8)$ und ChristoPHERSEN (10) finden allerdings auch beim Rattenleberenzym bis zu hohen GSH-Konzentrationen eine Kinetik erster Ordnung. $\mathrm{Da}$ es zu klären galt, ob diese Diskrepanzen auf eventuelle Verunreinigungen in den verwendeten Glutathionpräparaten oder methodische Unterschiede zurückzuführen sind, haben wir in vorliegenden Untersuchungen die Abhängigkeit der Reaktionsrate von der GSH-Konzentration mit 4 verschiedenen kommerziellen Präparaten durchgeführt und die Untersuchungen auf GSH-Konzentrationsbereiche ausgedehnt, die eindeutig oberhalb der angegebenen $\mathrm{K}_{\mathrm{m}^{-}}$ Werte liegen $(12,18)$.

Wir erhalten für beide Enzymchargen eine Kinetik erster Ordnung in bezug auf GSH in allen Konzentrationsbereichen, dementsprechend eine lineare Abhängigkeit der Reaktionsrate von der GSH-Konzentration bis zu $16 \mathrm{~mm}$ (unabhängig vom verwendeten GSHPräparat und der Enzymkonzentration). Abbildung 1 zeigt ein v/s Diagramm bei mäßigen GSH-Konzentrationen und geringen Enzymaktivitäten, Abbildung 2 analoge Kurven bei einer etwa 10 fachen Enzymkonzentration und hohen GSH-Konzentrationen. Die Ergebnisse von Hochstern und UTLEy (12) und DemusOoLe und Mitarbeitern (18) sind mit diesen Daten unvereinbar. Möglicherweise muß das Testsystem von Hochstern und Utrey (12) (gekoppelte Reduktion des gebildeten GSSG durch Glutathionreduktase) für die abweichenden Resultate verantwortlich gemacht werden. Wir konnten zeigen, daß aufgrund der Produkt-(GSH)Hemmung der Glutathionreduktase der gekoppelte Test $(12,29)$ bei hohen GSH-Konzentrationen unzuverlässig ist (31).

Eine Abschätzung der Michaeliskonstante für $\mathrm{H}_{2} \mathrm{O}_{2}$ nach 1. c. (14) bei $0,1 \mathrm{~mm}$ GSH und $37^{\circ}$ ergab für Blut- 


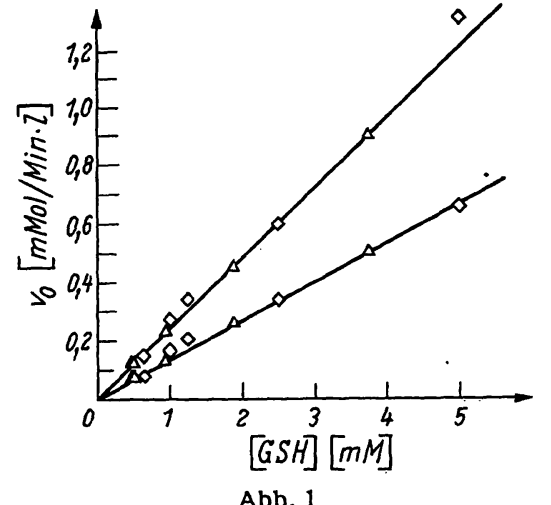

Abb. 1

Abhängigkeit der enzymatischen $\mathrm{GSH}-\mathrm{Oxydation}$ durch $\mathrm{H}_{2} \mathrm{O}_{2}$ von der GSH-Konzentration.

Bedingungen: $0,125 \mathrm{M}$ K-Phosphatpuffer $\mathrm{pH} \mathrm{7,0;0,25} \mathrm{mM} \mathrm{EDTA;}$ $37^{\circ} ; \mathrm{H}_{2} \mathrm{O}_{2}$ to $: 1,25 \mathrm{mM}$. $\mathrm{v}_{0}$ ist angegeben in mMol/Min. und $l$ Inkubaonsansatz. $\mathrm{S}$ in $\mathrm{mM}$ GSH.

- $-0-$ Glutathion von Merck

- Glutathion von Zellstoffwerk Waldhof

Obere Gerade: Glutathionperoxidase aus Lebergewebe $(0,13 \mathrm{U} / \mathrm{ml}$

untere Gerade: Glutathionperoxidase aus Erythrocyten $(0,07 \mathrm{U} / \mathrm{ml}$ Ansatz)
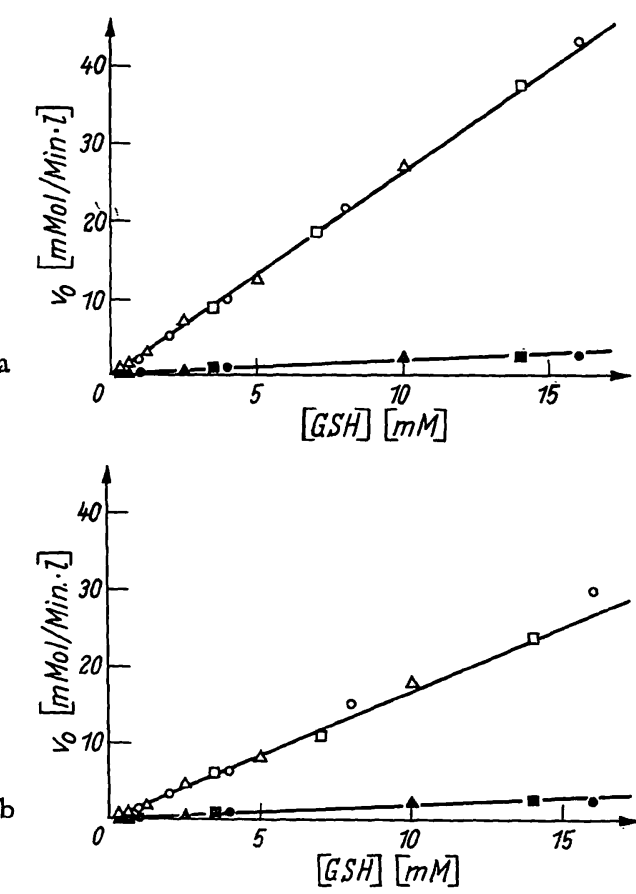

Abb. 2

Abhängigkeit der enzymatischen GSH-Oxydation (leere Symbole) durch $\mathrm{H}_{2} \mathrm{O}_{2}$ von der $\mathrm{GSH}-$ Konzentration.

Bedingungen, Geschwindigkeits- und Konzentrationsangaben wie in Abb. 1

- - GSH von Hoffmann La-Roche - $\square-\square-$ GSH von Boehringer

Die gefüllten Symbole bezeichnen die spontane Reaktionsrate.

a: Glutathionperoxidase aus Leber $(1,2 \mathrm{U} / \mathrm{ml}$ Ansatz);

b: Glutathionperoxidase aus Erythrocyten $(0,68 \mathrm{U} / \mathrm{ml}$ Ansatz)

und Leberenzym einen innerhalb der Fehlergrenze übereinstimmenden Wert von 2-3 $\mu$ m. Da die Bedingungen, unter denen Demus-Oole und Mitarbeiter einen $\mathrm{K}_{\mathrm{m}}$ $\left(\mathrm{H}_{2} \mathrm{O}_{2}\right)$-Wert von $6,6 \mu \mathrm{M}$ bei der Rattenleber-GSHPeroxidase fanden, noch nicht publiziert wurden und eine Abhängigkeit des $\mathrm{K}_{\mathrm{m}}\left(\mathrm{H}_{2} \mathrm{O}_{2}\right)$-Wertes von der Donorkonzentration mit hoher Wahrscheinlichkeit anzunehmen ist $(14,15)$, können unsere Ergebnisse mit denen von Demus-Oole und Mitarbeitern (18) nicht verglichen werden.

\section{Substratspezifität}

Wie die Reaktion der GSH-POD mit Glutathion, so verläuft auch der Umsatz von anderen SH-Verbindungen nach einer Kinetik 1. Ordnung. Wie aus Abbildung 3 $\mathrm{zu}$ ersehen ist, ist die in Wirklichkeit komplexere Spontanreaktion der SH-Verbindungen mit $\mathrm{H}_{2} \mathrm{O}_{2}$ im Anfangsbereich zumindest von einer Kinetik erster Ordnung nicht $\mathrm{zu}$ unterscheiden. Wir nahmen daher den

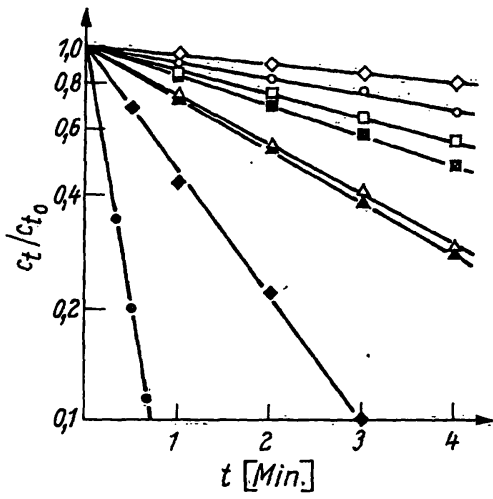

Abb. 3

Oxydation verschiedener $\mathrm{SH}-$ Verbindungen durch $\mathrm{H}_{2} \mathrm{O}_{2}$ in Abwesenheit (leere Symbole) und Anwesenheit (gefüllte Symbole) von Glutathionperoxidase aus Rattenleber. Die Ausgangskonzentration von RSH cto betrug $1 \mathrm{mM}$, die $\mathrm{H}_{2} \mathrm{O}_{2}$-Konzentration $1,25 \mathrm{~mm}$. Die weiteren Bedingungen: $0,125 \mathrm{~m} \mathrm{~K}$-Phosphatpuffer $\mathrm{pH} 7,0 ; 0,25 \mathrm{~mm}$ EDTA, 37

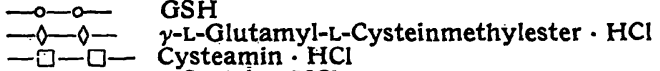

$$
\begin{aligned}
& \text { - } \triangle-\square-\text { Cysteamin } \cdot \mathrm{HCl}
\end{aligned}
$$

Abstand der mit und ohne Enzym erhaltenen Geraden aus Abbildung 3 als $\mathrm{Maß}$ für den zu gegebener Zeit erfolgten enzymatischen Umsatz.

Tabelle 3 zeigt die Aktivitäten von Leber- und Erythrocyten-Glutathionperoxidase in Peroxidaseeinheiten ( $\Delta \mathrm{log}$

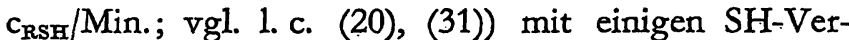
bindungen bezogen auf eine Aktivität mit GSH $=100 \%$.

Tab. 3

Relative Umsatzraten der Glutathionperoxidase aus Erythrocyten und Lebergewebe mit verschiedenen Donorsubstraten

\begin{tabular}{lcc}
\hline Donorsubstrat & $\begin{array}{c}\text { GSH-Peroxidase } \\
\text { aus Erythrocyten }\end{array}$ & $\begin{array}{r}\text { GSH-Peroxidase } \\
\text { aus Lebergewebe }\end{array}$ \\
\hline 1. Glutathion & $100,0 \%$ & $100,0 \%$ \\
2. $\gamma$-L-Glutamyl-L- & $30,0 \%$ & $23,0 \%$ \\
Cysteinmethylester - HCl & $10,0 \%$ & $11,5 \%$ \\
3. Mercaptoessigsäure- & $1,4 \%$ & $1,0 \%$ \\
methylester & $1,0 \%$ & $1,0 \%$ \\
5. Cysteamin - HCl & $0,7 \%$ & $0,9 \%$ \\
6. $\beta$-Mercaptein - HCl & $1,4 \%$ & $1,8 \%$ \\
7. $\beta$-Mercaptopropionsäure & methylester &
\end{tabular}

Sofern ausreichende Enzymaktivitäten eingesetzt werden, finden wir im Gegensatz zu MrLus (19) mit allen untersuchten SH-Verbindungen signifikante Ümsetzungen. Allerdings entsprechen die enzymatischen Umsatzraten mit Cystein und Cysteamin bei weitem nicht denen, die LITTLE und O'BRIEN mit dem Linolsäurehydroperoxid-RSH-System ( 30\% des Glutathionumsatzes) erhielten (8).

Obwohl die Reaktivität der Peroxidase mit den verschiedenen SH-Verbindungen ein breites Spektrum überstreicht, liegen die relativen Aktivitäten beim Blut- und 
Leberenzym in der gleichen Größenordnung. Die geringfügigen Differenzen dürften eher auf unvermeidbare Unzulänglichkeiten des Auswertungsmodus als auf unterschiedliche Affinität der Enzyme zu den jeweiligen Substraten zurückzuführen zu sein. (Die Untersuchungen wurden bei unterschiedlicher Enzymaktivität durchgeführt; das Verhältnis von enzymatischer zu spontaner Reaktionsrate fällt aber besonders bei Substanzen mit hoher Spontanreaktion stark ins Gewicht, wenn keine der konkurrierenden Reaktionen nach einer Kinetik nullter Ordnung verläuft. Die von uns vorgenommene Auswertung stellt also nur eine relativ grobe Abschätzung der enzymatischen Reaktionsraten dar.)

\section{Elektrophorese}

Um außer den funktionellen wenigstens noch einen chemischen Identitätsnachweis hinzufügen zu können, wurde das elektrophoretische Verhalten der Enzyme auf Cellogelstreifen geprüft. Die von uns ausgearbeitete Darstellung der GSH-POD auf Elektrophoresestreifen gestattet die Lokalisierung des Enzyms aufgrund seiner enzymatischen Eigenschaft und ist somit auch bei uneinheitlichen Präparaten verläßlich (25). Bei insgesamt 17 Elektrophoreseläufen unter verschiedenen Bedingungen ( $\mathrm{pH} 5,5-8,0 ; 3$ verschiedene Ionenstärken) fand sich keinerlei Unterschied in der elektrophoretischen Beweglichkeit von Erythrocyten- und Leberenzym.

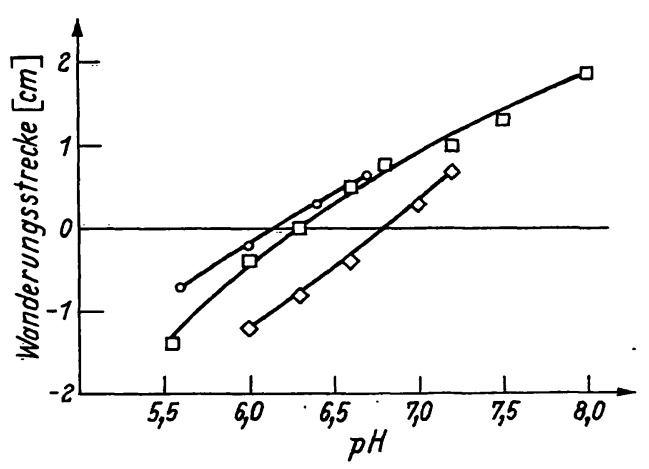

Abb. 4

Elektrophoretische Beweglichkeit der Glutathionperoxidase der Ratte in der BECKMAN-Mikrozonenelektrophorese in Abhängigkeit vom $\mathrm{pH}$. Da keine Unterschiede żwischen dem Leber- und Erythrocytenenzy
festgestellt wurden, ist jeweils nur ein Meßpunkt eingetragen. Versuchsbedingungen:

-o- - Tris-Maleat-Puffer $(I=0,0442), 200 \mathrm{~V}, 2-3 \mathrm{~mA}$

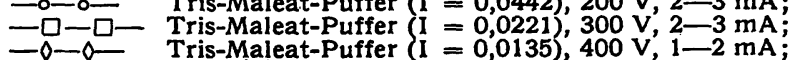
Laufzeit jeweils $1 \mathrm{Std}$. Die I-Werte geben die Ionenstärke beim IP an

Wie Abbildung 4 zeigt, erhalten wir unter Berücksichtigung der in l. c. (25) genannten Einschränkungen für Glutathionperoxidase der Ratte IP-Werte von $6,1,6,3$ und 6,8 bei $I=0,014,0,022$ und 0,044 .

\section{Diskussion}

Für die Identität von Glutathionperoxidase der Leber (C-Faktor I) und der von Mrrus (2) erstmals beschriebenen Glutathionperoxidase aus Erythrocyten. führten Neubert, Wojtczax und Lehninger (1) folgende Hinweise an:
1. C-Faktor I zeigt eine hohe spezifische GSH-PODAktivität.

2. Eine Präparation von GSH-POD aus Erythrocyten nach MrLls zeigt C-Faktor-Aktivität.

3. GSH-POD aus Erythrocyten (angereichert nach MrLLs) und C-Faktor I sind labil.

4. GSH-POD aus Erythrocyten und C-Faktor I verhalten sich ähnlich auf DEAE-Cellulose.

Argumente, die für eine Organspezifität der GSH-POD sprachen $(8,12,17,18)$, konnten durch vorliegende Untersuchungen entkräftet werden:

1. Stabilitätsunterschiede der gereinigten Enzyme konnten nicht festgestellt werden.

2. Das Enzym zeigt in der Leber und im Blut das gleiche kinetische Verhalten.

3. Es wurden kcine signifikanten Unterschiede in der Reaktivität mit Fremdsubstraten beobachtet.

Als weiterer Hinweis auf eine nicht nur funktionelle Identität der GSH-POD aus Blut und Leber kann das übereinstimmende chromatographische Verhalten der Enzyme auf Sephadex G 150 und ihre gleiche elektrophoretische Beweglichkeit aufgeführt werden.

Nachdem also an einer funktionellen und wahrscheinlich auch chemischen Identität der GSH-POD aus Leber und Erythrocyten kaum Zweifel möglich sind, können wir die mit dem Leberenzym gesammelten Erfahrungen (1, 7-11) zur Interpretation der biologischen Funktion der GSH-POD in den Erythrocyten und der Pathogenese enzymopenischer Anämie nutzbar machen.

Wir können mit Sicherheit annehmen, daß der GSHPOD in den Erythrocyten neben der Katalase eine bedeutende Rolle bei der Beseitigung von $\mathrm{H}_{2} \mathrm{O}_{2}$-Spuren zukommt (13-15, 20). Gegen diese von Milis (2) aufgestellte und von CoHEN und HochsteIN (13) weiterentwickelte Theorie wurde von AEBr, Heiniger und Lauber (32) geltend gemacht, daß die Peroxidaseaktivität der Katalase vernachlässigt werde. Diesem Argument kommt nach Angaben von Nicholls (33) jedoch keine entscheidende Bedeutung $z u$, da in Erythrocyten zumindest kein Donor für die Katalase von ausreichender Reaktivität in genügender Konzentration nachzuweisen ist. Die theoretischen Überlegungen von Nicholls (33), wonach allein die Katalase, und zwar in ihrer katalatischen Funktion für die $\mathrm{H}_{2} \mathrm{O}_{2}$-Elimination in Erythrocyten verantwortlich ist, konnten unlängst von FLOHÉ und BrAND widerlegt werden $(14,15)$.

Von vielen Autoren (13-15, 20, 29, 32, 34-37) wird weiterhin diskutiert, $\mathrm{da} \beta$ die pathophysiologischen Symptome bei verschiedenen Typen enzymopenischer hämolytischer Anämien letztlich durch den Ausfall der GSH-Peroxidasereaktion bedingt sind. Für dieses Konzept spricht vor allem die Ähnlichkeit der pathologischen Phänomene (Neigung zu hämolytischen Krisen, Bildung von HEINzschen Innenkörpern, verkürzte Lebensdauer der Erythrocyten, Reticulocytose, verminderter GSH-Gehalt oder Instabilität des GSHSpiegels in den Erythrocyten, eventuell Ikterus), die bei 
verschiedenartigen Enzymdefekten wie Glucose-6-Phosphatdehydrogenase- (38-47) und GSH-Reduktasemangel $(37,42)$ oder Störungen der Glutathionsynthese (42-44) beobachtet werden können. Diese Enzymdefekte zeichnen sich letztlich durch eine Gemeinsamkeit aus: die verminderte Kapazität zur Bereitstellung reduzierten Glutathions in der Zelle (37-44). Dem entspricht aufgrund der kinetischen Eigenschaften der Glutathionperoxidase (s. o. und 1. c. (7), (8), (10), (13-15), (20)) eine geringere Peroxidelimination durch GSH. Diese Interpretation der pathophysiologischen Vorgänge bei den erwähnten Enzymdefekten wird weiterhin durch kürzlich publizierte Beobachtungen von Borvin und Mitarbeitern (45) und Necheles und Mitarbeitern $(46,47)$ gestützt: Die von den Autoren beschriebenen Patienten mit einem isolierten Mangel an GSH-POD in den Erythrocyten zeigen nahezu die gleichen klinischen Symptome, die uns von den schon länger bekannten enzymopenischen hämolytischen Anämien her bekannt sind. In diesem Zusammenhang ist auch die erhöhte Empfindlichkeit von fetalen Erythrocyten gegenüber hämolysierenden Agentien zu erwähnen. Wahrscheinlich muß sie im Zusammenhang mit der ausgeprägtẹp Altersabhängigkeit des GSH-POD-Spiegels $(18,48-50,57)$ verstanden werden.

Zweifellos ist es also die Glutathionperoxidase, die die Erythrocyten vor oxidativer Zellschädigung bewahrt. Weniger durchsichtig ist allerdings, wodurch unmittelbar die pathophysiologischen Symptome bei GSHMangelzuständen oder niedrigerem GSH-POD-Spiegel bedingt sind. Zur Zeit gilt als wahrscheinlichste Hypothese, daß es sich hierbei um toxische Effekte von $\mathrm{H}_{2} \mathrm{O}_{2}$ handelt. Als Angriffspunkte des Wasserstoffperoxids werden Zellmembran, Hämoglobin oder Enzyme diskutiert. Dafür sprechen u. a. Befunde von CoHEN und HochsteIN (34), die $\mathrm{H}_{2} \mathrm{O}_{2}$-Bildung in den Erythrocyten bei Behandlung mit Hämolyse provozierenden Substanzen (Phenylhydrazin, Menadion, Primachin und Hydrochinon) nachweisen konnten. Zudem fanden LIEBow'tTz und Cohen (36) in Glucose-6-phosphathydrogenase-Mangel-Erythrocyten bei Inkubation mit Acetylphenylhydrazin höhere $\mathrm{H}_{2} \mathrm{O}_{2}$-Werte als in Normalzellen. Auch nach unseren Ergebnissen $(14,15) \mathrm{mu}$ bei einer unzureichenden Funktion der GSH-POD mit einer Akkumulation von $\mathrm{H}_{2} \mathrm{O}_{2}$ in den Erythrocyten gerechnet werden.

Die für das Verständnis der biologischen Funktion der GSH-POD grundlegenden Untersuchungen von CoHEN und Hochstein (13) weichen jedoch in einem Punkt entscheidend von dem pathophysiologischen Vorgang, den sie imitieren wollen, der oxidativen Zellschädigung, ab: Bei der Diffusion von $\mathrm{H}_{2} \mathrm{O}_{2}$ in eine Erythrocytensuspension bildet sich nach Abfall von GSH zunächst Methämoglobin, später tritt dann auch Hämolyse ein. Eine hämolytische Krise in vivo ist jedoch in der Regel nicht von einer Methämoglobinämie begleitet $(38-44,52)$. Auffällig ist weiterhin, daß sich das Hämoglobin in Erythrocyten durch Nitrit quantitativ in Methämoglobin überführen läßt, ohne daß es zwangsläufig zu einer Hämolyse kommt (53). Diese Oxidation von Hämoglobin durch Nitrit ist zudem nach CoHEN und HocHSTEIN ebenfalls von einer mäßigen $\mathrm{H}_{2} \mathrm{O}_{2}$-Bildung begleitet (35). Wir neigen daher zu der Annahme, daß weder ein exzessives Angebot von Oxidationsäquivalenten noch eine Akkumulation von $\mathrm{H}_{2} \mathrm{O}_{2}$ die Zerstörung der Erythrocytenmembran in vivo ursächlich erklären kann. Vielmehr möchten wir die Vermutung äußern, daß an der Pathogenese der Hämolyse andere Peroxide, z. B. Peroxide der in der Erythrocytenmembran reichlich vorhandenen ungesättigten Fettsäuren (54), beteiligt sind. Es ist leicht vorzustellen, daß neben $\mathrm{H}_{2} \mathrm{O}_{2}$ auch Lipidperoxide bei radikalisch verlaufenden Autoxidationsprozessen oder bei Einwirkung ionisierender Strahlen in der Erythrocytenmembran gebildet werden.

Die Glutathionperoxidase ist, wie erwähnt, wenig spezifisch in bezug auf ihr Peroxidsubstrat. Sie katalysiert mit vergleichbarer Geschwindigkeit die Reduktion von $\mathrm{H}_{2} \mathrm{O}_{2}$, t-Butylhydroperoxid (8), Linolsäurehydroperoxid (7-1.0, 16), Linolsäurehydroperoxidäthylester (8), Cumolhydroperoxid (8), Linolensäurehydroperoxid (11) schließlich sogar Thymin- und Nucleinsäurehydroperoxide (55). Dieses breite Spezifitätsspektrum, das die GSH-POD vor allen anderen Peroxiden auszeichnet, fand unseres Erachtens bisher bei der Diskussion der Enzymfunktion in den Erythrocyten zu wenig Beachtung. Unter Berücksichtigung der für den Leberstoffwechsel gesicherten Funktionen der Glutathionperoxidase ist vielleicht ein eingehenderes Verständnis der Entstehung hämolytischer Prozesse und eine Klärung der Wirkungsweise hämolysierender Substanzen zu erwarten.

Herm Prof. Dr. F. SCHNEIDER sei für sein Interesse an der Untersuchung und wertvolle Diskussionen und Frau I. MülLER für technische Hilfe gedankt. Die Arbeit wurde durch eine Sachbeihilfe der Deutschen Forschungsgemeinschaft unterstützt.

\section{Literatur}

1. Neubert, D., A.B. Wojtczak und A. L. Lehninger, Proc. Natl. Acad. Sci. USA 48, 1651 (1962). - 2. Mrles, G. C., J. biol. Chemistry 229, 189 (1957). - 3. Hunter Jr., F. E., A. Scotr, P. E. Hoffsten, F. Guerra, J. Weinstein, A. Schneider, B. Schutz, J. Fink, L. Ford und E. SMITth, J. biol. Chemistry 239, 604 (1964). - 4. Hunter Jr., F. E., A. Scott, P. E. HoffSten, J. M. Gebicki, J. Weinstein und A. Schneider, J. biol. Chemistry 239, 614 (1964). - 5. Hunter Jr., F. E., A. Scort, J. Weinstein und A. Schneider, J. biol. Chemistry 239, 622 (1964). - 6. Gebicki, J. M. und F. E. Hunter Jr., J. biol.
Chemistry 239, 631 (1964). - 7. O'Brien, P. J. und .C. LitTtLe, Biochem. J. 103, 31 p (1967). - 8. LITTLE, C. und P. J. O'BRIEN, Biochem. biophys. Res. Commun. 31, 145 (1968). - 9. O'Brien, P. J. und C. Lrtrle, Can. J. Biochem. 47, 493 (1969). - 10. ChrIstophersen, B. O., Biochim. biophysica Acta (Amsterdam) 164, 35 (1968). - 11. Chiristophersen, B. O., Biochim. biophysica Acta (Amsterdam) 176, 463 (1969). - 12. Hochsters, P. und H. Utrex, Mol. Pharmacol. 4, 574 (1968). - 13. Cohen, G. und P. Hochstein, Biochemistry (USA) 2, 1420 (1963). - 14. FLOHÉ, L. und I. Brand, Biochim. biophysica Acta (Amsterdam) 191, 
541 (1969). - - 15. FlortE, L., Hoppe-Seylers Zschr. physiol. Chem. 350, 1181 (1969). - 16. O'BrIEN, P. J. (persönliche Mitteilung). - 17. Neubert, D., T. H. Rose und A. L. Lehninger, J. biol. Chemistry 237, 2025 (1962). - 18. Demus-Oole, A. M., E. Swierczewski und A. Minkowski, diese Z. 7, 209 (1969). - 19. Mills, G. C., J. biol. Chemistry 234, 502 (1959). - 20. SChNEIDER, F. und L. FloHÉ, Hoppe-Seylers Zschr. physiol. Chem. 348, 540 (1967). - 21. Benorron, L., Canad. J. Chem. 40, 570 (1962). - 22. Roeske, R., J. org. Chemistry 28 , 1251 (1963). - 23. Schnabel, E., Liebigs Ann. Chem. 702, 188 (1967). - 24. Fischer, E. und U. Suzukr, Hoppe-Seylers Zschr. physiol. Chem. 45, 405 (1905). - 25. Fuohé, L., Hoppe-Seylers Zschr. physiol. Chem. 350, 856 (1969). - 26. Lowry, O. H., N. J. Rosebrough, A. L. FARr und R. J. RANDall, J. biol. Chemistry 193, 265 (1951). - 27. Thauer, R. K., A. Meiforth und H. UeiLEkE, Arch. exper. Path. Pharmak. 252, 291 (1965). - 28. Aegr, H. E., Radiation Research, Supplement 3, 130 (1963). - 29. Paglia, D. E. und W. N. Valentrne, J. Laborat. Clin. Med. S. Louis 70, 158 (1967). - 30. O'Brien, P. J., Can. J. Biochem. 47, 485 (1969). - 31. Floré, L. und I. Brand diese Z. 8, 156 (1970). 32. AEBi, H., J. P. Heiniger und E. Lauber, Helv. Chim. Acta 47, 1428 (1964). - 33. Nicholls, P., Biochim. biophysica Acta (Amsterdam) 99, 286 (1965). - 34. Corren, G. und P. Hochstein, Biochemistry (USA) 3, 895 (1964). 35. Cohen, G., M. Martinez und P. Hochstern, Biochemistry (USA) 3, 901 (1964). - 36. Liebowitz, J. und G. Conen, Biochem. Pharmacology 17, 983 (1968). - 37.
Waller, H. D., H. Chr. Benorhr und P. Waumans, Klin. Wschr. 47, 25 (1969). - 38. Carson, P. E., C. L. Flanagan, C. E. Ickes und A. S. Alving, Science (Washington) 124, 484 (1956). - 39. Carson, P. E., S. L. Schrier und A. S. Alving, J. Laborat. Clin. Mcd.S. Louis 48, 794 (1956). - 40. LörIR, G. W. und H. D. WALLER, Dtsch. med. Wschr. 86, 27, 87 (1961). - 41. Beutler, E., C. K. Mathai und J. E. Smith, Blood 31, 131 (1968). - 42. WalLER, H. D. und H. D. SCHoEN, Klin. Wschr. 44, 189 (1966). - 43. Prins, H. K., M. Oort, I. A. Loos, C. Zürcher und T. BeCKers, Blood 27, 145 (1966). - 44. Borvin, P. und C. Galand, Nouvelle Rev. Franc. Hematol. 5, 707 (1965). - 45. Borvin, P., C. GaLAND, J. HAKIM, J. RogÉ und N. Guéroult, Enzymol. biol. clin. 10, 68 (1969). - 46. Necheles, T. F., T. A. Boles und D. M. Allen, Blood 28, 988 (1966). - 47. Necheles, T. F., N. Maldonado, A. Barquet-Chediak und D. M. Allen, Blood 30, 880 (1967). 48. Gross, R. T., R. Bracci, N. Rudolph, E. SCHroeder und J. A. Kochen, Blood 29, 481 (1967). - 49. Pinto, R. E. und W. BARTLEY, Biochem. J. 112, 109 (1969). - 50. SwIERCZEWSKI, E., A. M. Demus-Oole und A. Minkowsky, diese Z. 7, 208 (1969). - 51. Butenandt, O. (persönliche Mitteilung). - 52. Brewer, G. J., A. R. TARLow, R. W. Krllermeyer und A. S. Alving, J. Laborat. Clin. Med. S. Louis 59, 905 (1962). 53. Harley, J.D. und H. Robin, Blood 20, 710 (1962). 54. Gercken, G. und U. BrockmanN, Eutop. J. Biochem. 8, 489 (1969). - 55. Chiristophiersen, B. O., Biochim. biophysica Acta (Amsterdam) 186, 387 (1969).
Dr. L. Flohé 74 Tübingen Hoppe-Seyler-Str. 1 